\title{
ON THE NATURE OF THE VARIABLE GAMMA-RAY SOURCES AT LOW GALACTIC LATITUDES
}

\author{
VALENTÍ BOSCH-RAMON ${ }^{1}$, GUSTAVO E. ROMERO ${ }^{2}$ and JOSEP M. PAREDES ${ }^{1}$ \\ ${ }^{1}$ Universitat de Barcelona, Spain; E-mail:vbosch@am.ub.es \\ ${ }^{2}$ Instituto Argentino de Radioastronomía, FCAGLP, Universidad Nacional de La Plata, Spain
}

(Received 2 June 2004; accepted 5 August 2004)

\begin{abstract}
Population studies of EGRET gamma-ray sources indicate that there is a distinctive population of bright sources at low galactic latitudes. The sources have a distribution consistent with that of young galactic objects, with a concentration toward the inner spiral arms. There is a subgroup that displays strong variability with timescales from days to months. Following an earlier suggestion by Kaufman Bernadó et al. (2002), we explore the possibility that these sources could be high-mass microquasars. Detailed models for the gamma-ray emission that include inverse Compton interactions of electrons in the relativistic jets and photons from all local fields (stellar UV photons, synchrotron photons, soft X-ray photons from the accretion disk, and hard X-ray photons from a corona) are presented. We conclude that microquasars are excellent candidates for the parent population of the subgroup of variable low-latitude EGRET sources.
\end{abstract}

Keywords: microquasars, X-ray binaries, jets, gamma-rays

\section{Introduction}

Population studies of EGRET gamma-ray sources indicate that there is a distinctive population of bright sources at low galactic latitudes (Gehrels et al., 2000; Grenier, 2001, in press; Romero, 2001). They are well correlated with star forming regions and HII regions, which is indicative of an association with young stellar objects (Romero et al., 1999; Romero, 2001). $\log N-\log S$ studies suggest that they are more abundant toward the inner spiral arms (Gehrels et al., 2000; Bhattacharya et al., 2003). These are bright sources (isotropic luminosities in the range $10^{34-36}$ $\mathrm{erg} \mathrm{s}^{-1}$ ), with an average photon spectral index $\langle\Gamma\rangle=2.18$ (Gehrels et al., 2000), where $F(E) \propto E^{-\Gamma}$. These sources, whose number is $\sim 45 \pm 9$, form the GammaRay Population I (GRP I, Romero et al., in press). Among GRP I sources there is a subgroup that displays significant variability on timescales of weeks to months (Torres et al., 2001; Nolan et al., 2003). Recently, Kaufman Bernadó et al. (2002) and Romero et al. (in press) have suggested that this subgroup of GRP I sources might be high-mass microquasars (i.e. microquasars formed by a compact object and an early-type stellar companion), where the gamma-ray emission arises from interactions between relativistic particles in the jet and external photon fields, most notably the stellar UV emission. 
In the present paper we explore in more detail this hypothesis, presenting more realistic models for the gamma-ray emission. In particular, we will include effects of the interaction of the microquasar jet with the X-ray fields produced by the accretion disk and the hot corona that is thought to surround the compact object. We will also include synchrotron self-Compton emission, Klein-Nishina effects, and the back-reaction of the different losses in the particle spectrum of the jet. We will calculate, for some representative sets of the parameters that characterize highmass microquasars, the spectral energy distribution (SED) from radio wavelengths up to $\mathrm{GeV}$ gamma-rays.

\section{GRP I Sources}

GRP I sources concentrate along the galactic plane and present a good spatial correlation with young stellar objects (Romero et al., 1999). The variability analysis of these sources by Torres et al. (2001) clearly shows evidence for the existence of a subgroup with variable emission on timescales from weeks to months. This is corroborated by the recent results presented by Nolan et al. (2003), which are based on a maximum likelihood re-analysis of the EGRET data. These authors identify 17 variable sources within $6^{\circ}$ from the galactic plane. These sources are clumped within $55^{\circ}$ of the galactic center.

A $\log N-\log S$ analysis for all GRP I sources yields a distribution that is consistent with a power-law with index $\langle\beta\rangle=3.1 \pm 0.4$ (Bhattacharya et al., 2003). This is far steeper than what is expected for a population uniformly distributed along the galactic disk. For instance, for pulsars detected at $400 \mathrm{MHz}$ the slope results $\langle\beta\rangle \sim 1.05$ (Bhattacharya et al., 2003). The unidentified gamma-ray sources, on the contrary, seem to be concentrated mainly in the inner spiral arms. In order to find possible further evidence for different populations among GRP I sources, we have implemented a $\log N-\log S$ analysis of both variable and non-variable low-latitude sources.

First we have considered the 17 variable sources listed by Nolan et al. (2003). In order to take into account systematic effects introduced by different exposure and background resulting in non-uniform detectability, we have adopted the procedure described by Reimer (2001). The obtained $\log N-\log S$ plot is shown in Figure 1. The normalized distribution can be fitted by a power-law $N(S) \propto S^{-\beta}$, with $\langle\beta\rangle=1.66 \pm 0.31$, significantly flatter than for the entire sample. If we now consider those sources classified as non-variable or dubious cases, we get the $\log N-$ $\log S$ plot shown in Figure 2. In this case the distribution can be fitted by a power-law with index $\langle\beta\rangle=2.92 \pm 0.36$. Quoted errors for $\beta$ are 1 sigma errors.

The average spectral index is also different for both samples: in the case of the variable sources we have $\langle\Gamma\rangle=2.04 \pm 0.1$, whereas for the remaining sources is $\langle\Gamma\rangle=2.16 \pm 0.2$. However, the averaged errors are still large, due to the low number of sources, and do not allow yet to distinguish both populations properly. 


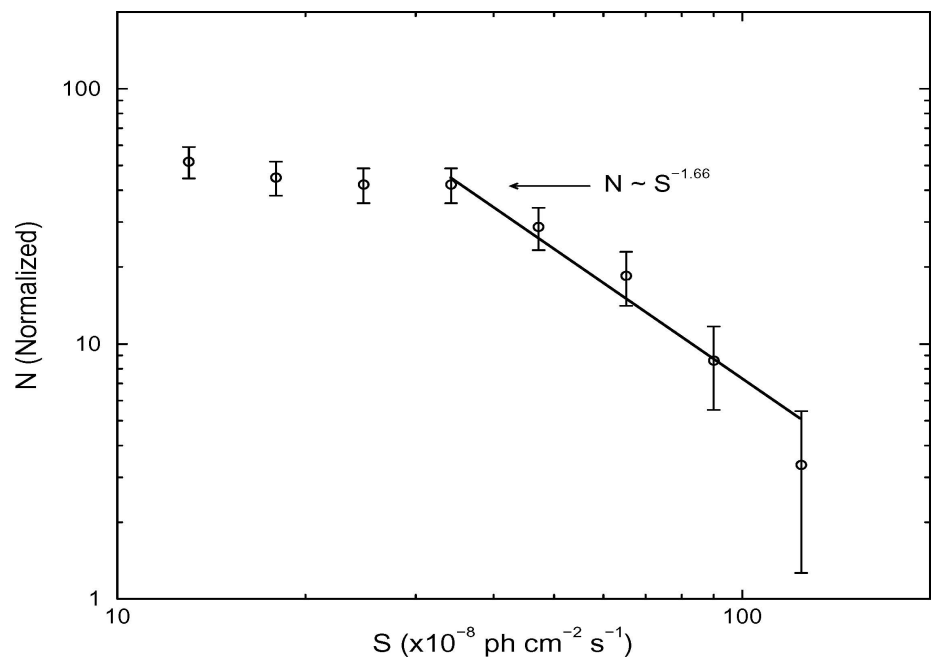

Figure 1. $\log N-\log S$ plot for variable gamma-ray sources within $|b|<6^{\circ}$.

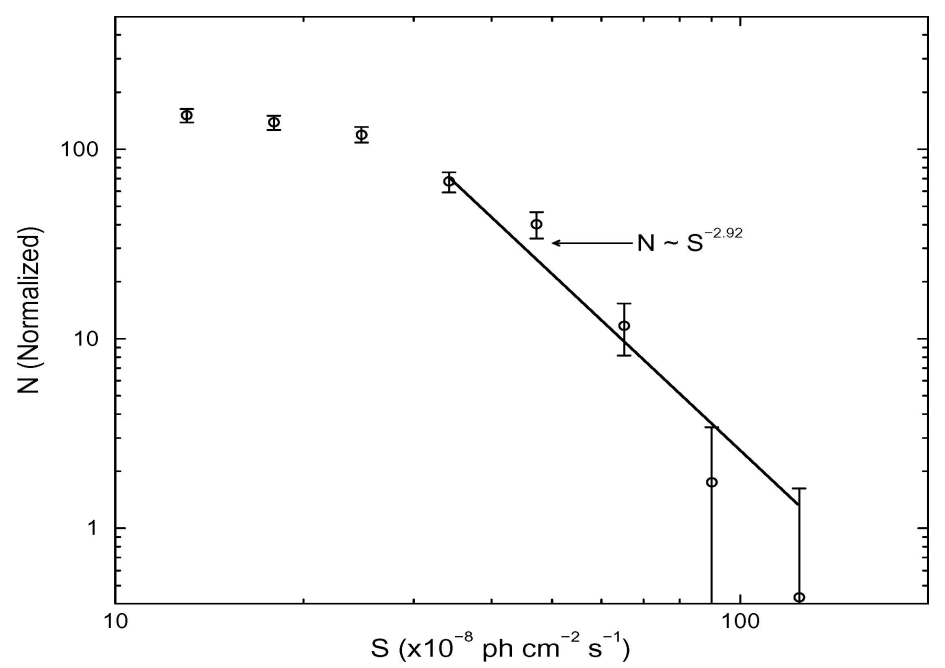

Figure 2. $\log N-\log S$ plot for non-variable gamma-ray sources within $|b|<6^{\circ}$.

In any case, $\log N-\log S$ analysis suggest that there are two different groups of sources, one formed by steady sources concentrated toward the inner spiral arms, and a second group with variable sources and a wider distribution along the galactic plane, although not as wide as that of radio pulsars (Bhattacharya et al., 2003).

High-mass microquasars appear to be good candidates for compact and variable sources in the galactic plane. Since they can have very large proper motions (e.g. Ribó et al., 2002), their distribution along the plane should be broader than that presented by supernova remnants and molecular clouds, which can be traced by star-forming regions and $\mathrm{OB}$ associations. Their spread, however, is limited by the 
lifespan of the companion massive star, and hence it is not as extended as that of radio pulsars. In the next section we will discuss the potential of microquasars as gamma-ray sources.

\section{Gamma-Ray Emission from Microquasars}

In the present paper we will consider a relativistic inhomogeneous jet flow moving along the $z$-axis (assumed to be perpendicular to the disk) with a bulk Lorentz factor $\Gamma_{\text {jet }}$. We shall allow the jet to expand laterally, in such a way that the radius $R$ at a distance $z$ from the compact object will be given by $R(z)=\xi z^{\varepsilon}$, with $\varepsilon \leq 1$ and $z_{0} \leq z \leq z_{\max }$. $\xi$ is related to the opening angle of the jet. For $\epsilon=1$ we have a conical jet. The electron energy distribution $\left(N\left(z, \gamma_{\mathrm{e}}\right)\right.$, a power-law of index $\left.p\right)$, the maximum electron Lorentz factor $\left(\gamma_{\mathrm{emax}}(z)\right)$ and the magnetic field $(B(z))$ will be parametrized like in Ghisellini et al. (1985).

The synchrotron radiation density within the jet can be estimated using the local approximation to the synchrotron radiation field (e.g. Ghisellini et al., 1985). We have used the formulae presented in Pacholczyk (1970), adapted to an expanding jet. The total radiation field to which the leptons are exposed in the jet will have also contribution from external sources. These contributions can be modeled as two black body components, one peaked at UV energies (the companion star field) and other at energies $k T \sim 1 \mathrm{keV}$ (the inner accretion disk field), plus a powerlaw with an exponential cutoff at $k T \sim 150 \mathrm{keV}$ (the corona). With the exception of the disk, these contributions are assumed to be isotropic (Romero et al., 2002; Georganopoulos et al., 2002).

Once $N\left(z, \gamma_{\mathrm{e}}\right)$ and the total energy density in seed photons $\left(U_{\mathrm{tot}}\left(\epsilon_{0}, z\right)\right)$ have been determined, the inverse Compton (IC) interaction between them can be studied calculating the IC spectral energy distribution per energy unit. The cross section $\left(\sigma\left(x, \epsilon_{0}, \gamma_{\mathrm{e}}\right)\right)$ for both the Thomson and the Klein-Nishina regimes has been taken from Blumenthal and Gould (1970). The spectral energy distribution for the optically thin case in the jet's reference frame is:

$$
\begin{aligned}
\epsilon L_{\epsilon}= & \epsilon \int_{z_{\min }}^{z_{\max }} \int_{\epsilon_{0 \min }(z)}^{\epsilon_{0 \max }(z)} \int_{\gamma_{\mathrm{emin}}(z)}^{\gamma_{\mathrm{emax}}(z)} \Sigma(z) U_{\mathrm{tot}}\left(\epsilon_{0}, z\right) \\
& \times N\left(\gamma_{\mathrm{e}}, z\right) \frac{d \sigma\left(x, \epsilon_{0}, \gamma_{\mathrm{e}}\right)}{d \epsilon} \frac{\epsilon}{\epsilon_{0}} d \gamma d \epsilon_{0} d z,
\end{aligned}
$$

where $\epsilon_{0}$ and $\epsilon$ are the energies of the incoming and the outgoing photons, respectively. The parameter $x$ is actually a function which depends on both of the former quantities and on the electron Lorentz factor. $\Sigma(z)$ is the surface of a perpendicular jet slice located at $z$. Notice that the external fields contributing to $U_{\text {tot }}\left(\epsilon_{0}, z\right)$ should be transformed to the co-moving frame. Detailed expressions for such transformations are given by Dermer and Schlickeiser (2002). 
In the observer's reference frame we have:

$$
\epsilon^{\prime} L_{\epsilon^{\prime}}^{\prime}=D^{2+p} \epsilon^{\prime} L_{\epsilon^{\prime}} .
$$

The integration is performed in the co-moving system and then the result is transformed to the observer's frame, hence the factor $D^{2+p}$, which is the Doppler boosting for a continuous jet. The energy of the scattered photons in the jet's reference frame $(\epsilon)$ is boosted to $\epsilon^{\prime}=D \epsilon$. The Doppler factor $D$ for the approaching jet is given by

$$
D=\frac{1}{\Gamma_{\text {jet }}(1-\beta \cos \theta)},
$$

where $\beta$ is the velocity of the jet in speed of light units and $\theta$ is the angle between the jet and the line of sight.

In the case of the IC interactions with disk photons, a factor $(1-\cos \theta)^{(p+1) / 2}$ must be introduced in Eq. (1) in order to take into account the fact that the photons come from behind the jet (Dermer et al., 1992).

In order to make any calculation of the IC emission of a given microquasar, we have to specify first the jet power. In this work we shall adopt the disk/jet coupling hypothesis formulated by Falcke and Biermann $(1995,1999)$, i.e. the jet power is proportional to the accretion rate:

$$
L_{\text {jet }}=q L_{\mathrm{ac}} .
$$

where $L_{\mathrm{ac}}=\dot{M} c^{2}$ is the accretion power onto the compact object and $q$ is a number $<1$. For a leptonic jet, as in our case, $q \ll 1$. The jet power determines the energy content of the electron energy distribution for a leptonic jet (see, e.g. Georganopoulos et al., 2002).

Although the model outlined above concerns primarily to the high energy emission range since it focuses on the IC radiation from the inner jet, the synchrotron formulae can be used to make predictions also at radio wavelengths. With this aim, we have developed an outer jet model appropriate for regions farther from the compact object. This outer jet model will fulfill most of the conditions of the former one, but it will require a different geometry because of the expected lower energy loss rate of the particles.

For further details about our model, see Bosch-Ramon et al. (in press).

\section{Specific Models}

In order to explore a plethora of situations, we have adopted a set of assumptions, which are summarized in Tables I. In Table II we list those parameters that were 


\section{TABLE I}

Main fixed parameters in the models

\begin{tabular}{ll}
\hline Parameter (symbol) & Value \\
\hline Black hole mass $\left(M_{\mathrm{bh}}\right)$ & $10 M_{\odot}$ \\
Gravitational radius $\left(R_{\mathrm{g}}\right)$ & $1.48 \times 10^{6} \mathrm{~cm}$ \\
Accretion luminosity $\left(L_{\mathrm{ac}}\right)$ & $10^{-8} M_{\odot} \mathrm{c}^{2} \mathrm{year}^{-1}$ \\
Stellar radius $\left(R_{*}\right)$ & $15 R_{\odot}$ \\
Stellar bolometric luminosity $\left(L_{*}\right)$ & $5 \times 10^{38} \mathrm{erg} \mathrm{s}^{-1}$ \\
Distance from jet's apex to the compact object $\left(z_{0}\right)$ & $50 R_{\mathrm{g}}$ \\
Initial jet radius $\left(R_{0}\right)$ & $0.1 z_{0}$ \\
Orbital radius $\left(R_{\text {orb }}\right)$ & $45 R_{\odot}$ \\
Peak energy of the disk $\left(k T_{\text {disk }}\right)$ & $1 \mathrm{keV}$ \\
Peak energy of the corona $\left(k T_{\text {cor }}\right)$ & $150 \mathrm{keV}$ \\
Peak energy of the star $\left(k T_{\text {star }}\right)$ & $10 \mathrm{eV}$ \\
Expansion coefficient of the inner jet $(\varepsilon)$ & 1 \\
Electron power-law index $(p)$ & 2 \\
Photon index for the corona $\left(\Gamma_{\text {cor }}\right)$ & 1.6 \\
\hline
\end{tabular}

TABLE II

Changing parameters in the different cases considered in the text

\begin{tabular}{ll}
\hline Parameter (symbol) & Value mild/extreme/realistic \\
\hline Corona luminosity $\left(L_{\text {cor }}\right)$ & $10^{35}, 10^{35}, 3 \times 10^{32} \mathrm{erg} \mathrm{s}^{-1}$ \\
Total disk luminosity $\left(L_{\text {disk }}\right)$ & $10^{37}, 10^{37}, 5 \times 10^{32} \mathrm{erg} \mathrm{s}^{-1}$ \\
Inner jet magnetic field $\left(B\left(z_{0}\right)\right)$ & $10,10,200 \mathrm{G}$ \\
Maximum electron Lorentz factor $\left(\gamma_{\operatorname{emax}}\left(z_{0}\right)\right)$ & $10^{4}, 10^{6}, 10^{4}$ \\
Bulk Lorentz factor of the jet $\left(\Gamma_{\text {jet }}\right)$ & $2.5,10,1.1$ \\
Viewing angle to jet's axis $\theta$ & $10^{\circ}, 1^{\circ}, 10^{\circ}$ \\
Disk/jet coupling constant $(q)$ & $10^{-4}, 10^{-4}, 10^{-3}$ \\
\hline
\end{tabular}

allowed to change along with the values adopted for three representative cases: a 'mild' microquasar, a microblazar, and a system similar to LS 5039 and LS I + 61 $303^{1}$.

In Figure 3, we show the whole SED of the 'mild' case. We plot here the spectra for all contributions to the emission, i.e. the outer jet, the seed photon sources, and the IC components (for outer jet parameters, see Table III). In Figure 4 we show a more extreme case, for a source with a high bulk Lorentz factor $\Gamma_{\text {jet }}=10$, a high-energy cutoff for electrons of $\gamma_{\mathrm{emax}}\left(z_{0}\right)=10^{6}$, and a small viewing angle $\theta=1^{\circ}$ (see Table II). Finally, we plot a SED similar (realistic case in Tables II and III) to the one presented by two microquasars that are considered as the 
TABLE III

Outer jet parameters (different cases considered in the text)

\begin{tabular}{ll}
\hline Parameter (symbol) & Value mild/extreme/realistic \\
\hline Expansion coefficient of the outer jet $(\varepsilon)$ & $0.2,0.3,0.2$ \\
Initial point of the outer jet $\left(z_{0}\right)$ & $500 R_{\mathrm{g}}, 5000 R_{\mathrm{g}}, 500 R_{\mathrm{g}}$ \\
Initial radius of the outer jet $\left(R_{0}\right)$ & $50 R_{\mathrm{g}}, 500 R_{\mathrm{g}}, 50 R_{\mathrm{g}}$ \\
(Computed) maximum length of the jet (few GHz) & $\sim 10^{16}, \sim 10^{17} \sim 10^{15} \mathrm{~cm}$ \\
Maximum electron Lorentz factor of the outer jet $\left(\gamma_{\mathrm{emax}}\right)$ & $10^{3}, 10^{4}, 10^{3}$ \\
Outer jet magnetic field $\left(B\left(z_{0}\right)\right)$ & $1,0.1,20 \mathrm{G}$ \\
Electron power-law index $(p)$ & 2 \\
\hline
\end{tabular}

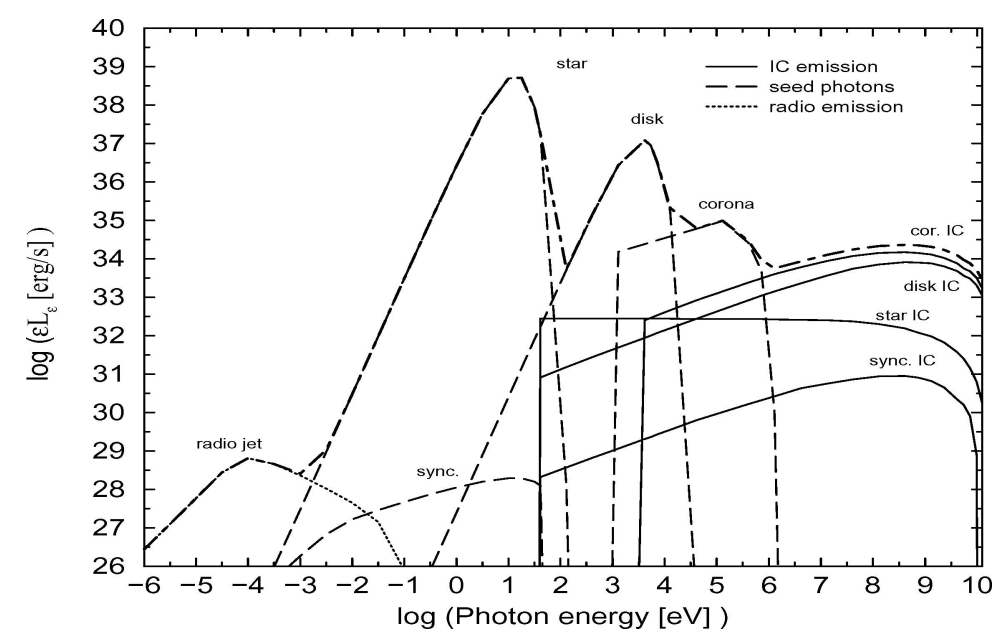

Figure 3. Complete SED for a model with $q=10^{-4}, B=10 \mathrm{G}, L_{\text {disk }}=10^{37} \mathrm{erg} \mathrm{s}^{-1}, L_{\text {cor }}=10^{35} \mathrm{erg}$ $\mathrm{s}^{-1}, \gamma_{\mathrm{emax}}\left(z_{0}\right)=10^{4}, \Gamma_{\text {jet }}=2.5$, and a viewing angle of $10^{\circ}$. The synchrotron seed photons come from the inner jet.

best candidates to counterparts of unidentified EGRET sources, LS 5039 and LSI $+61^{\circ} 303$ (3EG 1824+1514, Paredes et al., 2000, and 3EG $0241+6103$, Kniffen et al., 1997, respectively; see Figure 5); the selected set of parameter is consistent with those adopted in Bosch-Ramon and Paredes (2004a, in press).

\section{Discussion}

In a previous work (Bosch-Ramon et al., in press), it was shown that for several models with disk/jet coupling constant $q=10^{-3}$ we can get the expected luminosities in the observer's frame inferred for GRP I sources with the right photon index at energies $\sim 1 \mathrm{GeV}$, i.e. $\Gamma \sim 2$. When the magnetic field is strong enough 


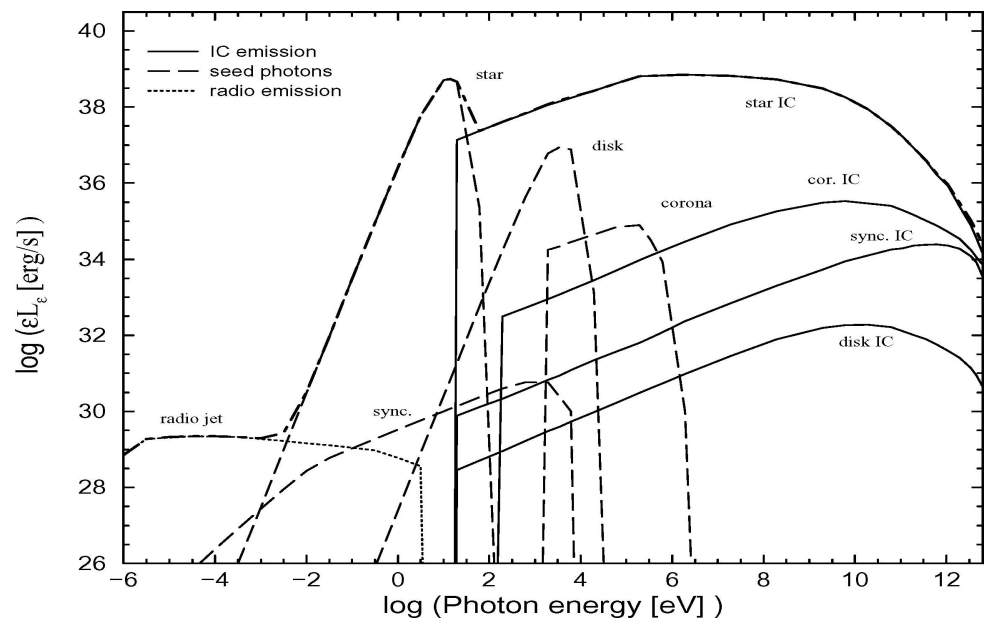

Figure 4. SED for a model with $q=10^{-4}, B=10 \mathrm{G}, L_{\text {disk }}=10^{37} \mathrm{erg} \mathrm{s}^{-1}, L_{\text {cor }}=10^{35} \mathrm{erg} \mathrm{s}^{-1}$, $\gamma_{\mathrm{emax}}\left(z_{0}\right)=10^{6}, \Gamma_{\mathrm{jet}}=10$, and a viewing angle of $1^{\circ}$. The synchrotron seed photons come from the inner jet.

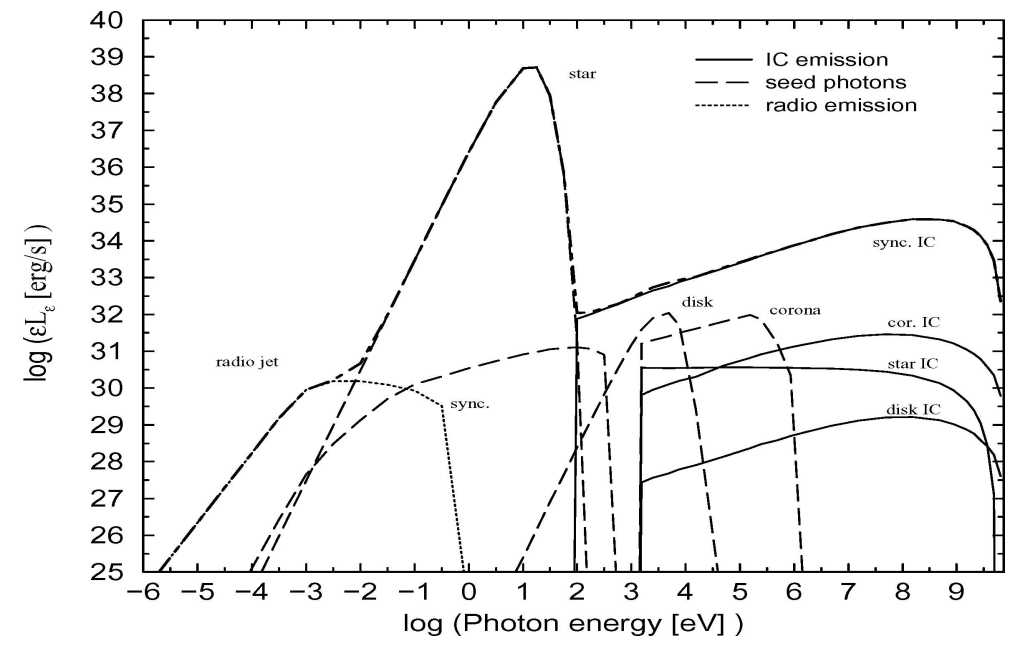

Figure 5. SED for a model with $q=10^{-3}, B=200 \mathrm{G}, L_{\text {cor }}$ and $L_{\text {disk }}=3 \times 10^{32} \mathrm{erg} \mathrm{s}^{-1}, \gamma_{\text {emax }}\left(z_{0}\right)=$ $10^{4}, \Gamma_{\text {jet }}=1.1$, and a viewing angle of $10^{\circ}$. The synchrotron seed photons come from the inner jet.

$\left(B\left(z_{0}\right) \sim 100 \mathrm{G}\right)$, SSC emission alone can account for luminosities $\sim 10^{35} \mathrm{erg} \mathrm{s}^{-1}$ at $1 \mathrm{GeV}$. Models with bulk Lorentz factors $\Gamma_{\text {jet }}=1.5$ and 5 do not produce dramatically different results for a jet with a viewing angle of $\sim 10^{\circ}$ except for the case of the scattered stellar photons. In case of lower magnetic fields, the IC scattering upon external fields clearly dominates. It is interesting to notice that even models with light jets $\left(q=10^{-5}\right)$ can produce significant gamma-ray sources $\left(\sim 10^{34} \mathrm{erg} \mathrm{s}^{-1}\right.$ at $100 \mathrm{MeV}$ ) when a strong corona is present. In these models, the spectrum tends to 
be a bit harder than in the case of stellar photons, with our current set of assumptions (value of $\Gamma_{\text {cor }}$, etc.).

In the present study of concrete cases, we compare Figures 3-5. From these figures, it is possible to see the differences between 'mild' microquasars, with $\Gamma_{\text {jet }}=2.5$, a viewing angle of $\theta=10^{\circ}$ and $q=10^{-4}$, and more 'extreme' cases with $\Gamma_{\text {jet }}=10$ and $\theta=1^{\circ}$. Since we have assumed in both cases a magnetic field of $B\left(z_{0}\right)=10 \mathrm{G}$, external IC scattering always dominates over SSC. In the 'mild' microquasar the emission at $\mathrm{MeV}-\mathrm{GeV}$ energies is mainly due to upscattering of coronal photons whereas, in the extreme case, the IC emission in the stellar field exceeds by far the other contributions. For the case similar to LS 5039/LSI $+61^{\circ} 303$, we have taken the magnetic field to be high enough to be the main contribution at high energies to reproduce the observed features of the spectra; mainly, higher luminosities at gamma-rays than at $\mathrm{X}$-rays and no strong disk/corona hints at $\mathrm{X}$-rays. In the three cases the radio counterpart is rather weak, with a $L_{\text {radio }} / L_{\gamma} \ll 1$. The hard X-ray counterpart, on the contrary, can be important, even beyond the cutoff for the corona (at $\sim 150 \mathrm{keV}$ ). Hence, INTEGRAL observations can be useful to unmask candidates that are obscured at optical wavelength (see Combi et al., 2004, for a recent study in this direction).

An interesting feature of the extreme case is that the gamma-ray emission with a hard spectrum $\Gamma \sim 2$ extends up to $\sim 100 \mathrm{GeV}$. Above $10^{11} \mathrm{eV}$ the spectrum becomes softer due to the Klein-Nishina effect. This type of sources should be detectable with modern Imaging Cherenkov Telescopes like HESS and MAGIC. Even systems with low-mass companions and weak coronas might be detectable since the SSC component has luminosities of $\sim 10^{35} \mathrm{erg} \mathrm{s}^{-1}$. The soft spectrum can be used to differentiate this leptonic model from other hadronic alternatives which predict a harder spectrum at very high energies (e.g. Romero et al., 2003).

\section{Conclusions}

We have shown that the variable gamma-ray sources found on the galactic plane have some common features that make reasonable to consider them as a distinctive group of GRP I sources. We have suggested that these sources might be microquasars with high-mass stellar companions and we have elaborated some detailed models to explain the gamma-ray production in this type of objects. In particular, we have considered inhomogeneous jet models where gamma-rays are the result of inverse Compton interactions of leptons in the inner jet with locally produced synchrotron photons as well as external photon fields. We have calculated the emission resulting from the upscattering of disk, coronal, and stellar photons, incorporating a full Klein-Nishina calculation and the effect of losses in the particle spectrum. We have shown that different spectral energy distributions at high energies can be obtained from different and reasonable combinations of the physical parameters such as magnetic field, jet power, coronal and disk luminosities, etc. The model 
can compute with a reasonable set of parameters a realistic SED, reproducing the ones associated to known microquasars. It seems to be clear that the microquasar phenomenon can be naturally extended up to the highest energies and that we can expect these objects to manifest themselves as a distinctive group of gamma-ray sources that might be detectable with satellite-borne instruments like those to be carried by AGILE and GLAST and even by ground-based Cherenkov telescopes like HESS and MAGIC.

Microquasars are not, of course, the only kind of galactic object that might display variable gamma-ray emission. Other alternatives include early-type binaries (Benaglia and Romero, 2003), accreting neutron stars (Romero et al., 2001), pulsar wind nebulae (Roberts et al., 2002) and exotic objects (Punsly et al., 2000). However, microquasars are perhaps the most attractive candidates to explain a significant fraction of the variable GRP I sources because of the well-established presence of relativistic jets in these objects, along with several external photon fields.

\section{Acknowledgements}

J.M.P. and V.B.-R. acknowledge partial support by DGI of the Ministerio de Ciencia y Tecnología (Spain) under grant AYA-2001-3092, as well as additional support from the European Regional Development Fund (ERDF/FEDER). During this work, V.B.-R. has been supported by the DGI of the Ministerio de Ciencia y Tecnología (Spain) under the fellowship FP-2001-2699. G.E.R. is supported by Fundación Antorchas and the Argentine Agencies CONICET and ANPCyT. This research has benefited from a cooperation agreement supported by Fundación Antorchas. We thank an anonymous referee by for constructive comments on manuscript.

\section{Note}

1. LS 5039 and $\mathrm{LSI}+61^{\circ} 303$ present significant differences though, regarding gamma-rays, they are pretty similar. Also, at lower energies, both systems can be treated in a similar way at this stage.

\section{References}

Benaglia, P. and Romero, G.E.: 2003, A\&A 399, 1121.

Bhattacharya, D., Akyüz, A., Miyagi, T., Samimi, J. and Zych, A.: 2003, A\&A 404, 163.

Blumenthal, G.R. and Gould, R.J.: 1970, Rev. Mod. Phys. 42, 237.

Bosch-Ramon, V. and Paredes, J.M.: 2004a, A\&A 417, 1075.

Bosch-Ramon, V. and Paredes, J.M.: 2004b, A\&A 425, 1069.

Bosch-Ramon, V., Romero, G.E. and Paredes, J.M.: 2005, A\&A 429, 267.

Combi, J.A., Ribó, M., Mirabel, I.F. and Sugizaki, M.: 2004, A\&A 422, 1031.

Dermer, C.D., Schlickeiser, R. and Mastichiadis, A.: 1992, A\&A 256, L27.

Dermer, C.D. and Schlickeiser, R.: 2002, ApJ 575, 667. 
Falcke, H. and Biermann, P.L.: 1995, A\&A 293, 665.

Falcke, H. and Biermann, P.L.: 1999, A\&A 342, 49.

Gehrels, N., Macomb, D.J., Bertsch, D.L., Thompson, D.J. and Hartman, R.C.: 2000, Nature 404, 363.

Georganopoulos, M., Aharonian, F.A. and Kirk, J.G.: 2002, A\&A 388, L25.

Ghisellini, G., Maraschi, L. and Treves, A.: 1985, A\&A 146, 204.

Grenier, I.A.: 2001, in: A. Carraminana, O. Reimer and D. Thompson (eds.), The Nature of Unindentified Galactic High-Energy Gamma-Ray Sources, Kluwer Academic Publishers, Dordrecht, p. 51.

Grenier, I.A.: 2004, in: Cosmic Gamma-Ray Sources, K.S. Cheng and G.E. Romero (eds.), Kluwer Academic Publishers, Dordrecht, p. 47.

Kaufman Bernadó, M.M., Romero, G.E. and Mirabel, I.F.: 2002, A\&A 385, L10.

Kniffen, D.A., Alberts, W.C.K., Bertsch, D.L. et al.: 1997, ApJ 486, 126.

Nolan, P.L., Tompkins, W.F., Grenier, I.A. and Michelson, P.F.: 2003, ApJ 597, 615.

Pacholczyk, A.G.: 1970, Radio Astrophysics, Freeman, San Francisco, CA.

Paredes, J.M., Martí, J., Ribó, M. and Massi, M.: 2000, Science 288, 2340.

Punsly, B., Romero, G.E., Torres, D.F. and Combi, J.A.: 2000, A\&A 364, 552.

Reimer, O.: 2001, in: A. Carramiñana, O. Reimer and D. Thompson (eds.), The Nature of Unindentified Galactic High-Energy Gamma-Ray Sources, Kluwer Academic Publishers, Dordrecht, p. 17.

Ribó, M., Paredes, J.M., Romero, G.E., Benaglia, P., Marti, J., Fors, O., Garcia-Sanchez, J.: 2002, $A \& A$ 384, 954.

Roberts, M.S.E., Gaensler, B.M. and Romani, R.W.: 2002, in: Patrick O. Slane and Bryan M. Gaensler (eds.), Neutron Stars in Supernova Remnants, ASP Conference Series, Vol. 271, ASP, San Francisco, 2002, p. 213.

Romero, G.E., Benaglia, P. and Torres, D.F.: 1999, A\&A 348, 868.

Romero, G.E.: 2001, in: A. Carramiñana, O. Reimer and D. Thompson (eds.), The Nature of Unindentified Galactic High-Energy Gamma-Ray Sources, Kluwer Academic Publishers, Dordrecht, p. 65.

Romero, G.E., Kaufman Bernadó, M.M., Combi, J.A. and Torres, D.F.: 2001, A\&A 376, 599.

Romero, G.E., Kaufman Bernadó, M.M. and Mirabel, I.F. 2002, A\&A 393, L61.

Romero, G.E., Torres, D.F., Kaufman Bernadó, M.M. and Mirabel, I.F.: 2003, A\&A 410, L1.

Romero, G.E., Grenier, I.A., Kaufman Bernadó, M.M. and Mirabel, I.F. and Torres, D.F.: in press, ESA-SP, [astro-ph/0402285].

Torres, D.F., Romero, G.E., Combi, J.A., Benaglia, P., Andernach, H. and Punsly, B.: 2001, A\&A 370, 468. 